\title{
PROKLA-Redaktion
}

\section{Editorial: Demokratie und Herrschaft, Parlamentarismus und Parteien}

Die große Krise hat erneut vor Augen geführt, wie prekär die Einrichtungen der parlamentarischen Demokratie angesichts einer mächtigen, global wirksamen kapitalistischen Dynamik sind. Sie selbst ist in der Krise. Regierungen werden auf Druck der EU und der Troika abgesetzt und durch Expertenregierungen ersetzt. Dem bundesdeutschen Parlament, das ohnehin viele Maßnahmen zur Bewältigung der Krise nur abnicken durfte, wurde von Bundeskanzlerin Merkel bescheinigt, dass es sich marktkonform zu verhalten habe. Die Krise hat nicht zu einer völligen Außerkraftsetzung der parlamentarischen Verfahren geführt, aber sie stark relativiert. Das gilt auf ganze andere Weise auch für den Prozess der europäischen Integration. Sie schwächt die Bedeutung der nationalstaatlichen demokratischen Institutionen, hat aber bislang kaum nennenswerten Ersatz dafür geboten. So stellt sich der Eindruck ein, dass eine Allianz von nationalen und europäischen Bürokraten in engem Kontakt mit den Unternehmensverbänden das Schicksal der EU bestimmt.

Solche für die Demokratie tatsächlich bedrohlichen Phänomene sollten nicht den Blick dafür verstellen, dass schon seit langem eine Erosion und Krise der Demokratie konstatiert wird. In der linken Diskussion stehen dafür die Namen Agnoli, Poulantzas, Hirsch und in jüngster Zeit Crouchs Diagnose von der Postdemokratie. Viele dieser Diagnosen lassen ein Unbehagen zurück. Zwar wurde immer wieder und mit plausiblen Argumenten auf Krisendynamiken und Erosionsprozesse hingewiesen. Das erweckt manchmal den Eindruck, dass in einer früheren Phase die parlamentarische Demokratie intakt war. Aber Zweifel sind angebracht. Gleichzeitig erwiesen sich in den vergangenen Jahrzehnten die parlamentarischen Institutionen als erstaunlich stabil. Sie erwiesen sich als stark genug, die Vertretungen von sozialen Bewegungen wie Grüne oder Linke aufzunehmen und sich auf diese Weise sogar zu erneuern.

Daraus ergeben sich Fragen danach, wie Krisen der Demokratie näher zu bestimmen sind. Wann kann man überhaupt von einer Krise der Demokratie sprechen? Was genau bedeuten die Krisenmomente innerhalb der parlamentarisch verfassten Politik? Kann es einen kontinuierlichen Abbau der Demokratie geben? In welchem Sinn kann davon gesprochen werden, dass die demokratischen Institutionen zu einem früheren Zeitpunkt nicht krisenhaft waren?

Der Nachweis einer Krise ist nicht leicht zu führen. Denn die Feststellung der Krise oder ihre Leugnung hängt auch von den Positionen ab, welche die jeweils Sprechenden vertreten. Dabei sind die Sprecherrollen gar nicht eindeutig festgelegt. Die Feststellung einer Krise der Demokratie kann auch von interessierten bürgerlichen Kräften oder von Rechtspopulisten kommen, die alles andere wollen, als ihre Verteidigung oder 
gar eine Demokratisierung der Demokratie selbst.

Die Schwierigkeiten hängen auch damit zusammen, dass die Kriterien für die Feststellung einer Krise der Demokratie nicht eindeutig sind. Momente der Krise sind Wahlfälschungen, die Störung des Wahlkampfs, die Begünstigung von bestimmten Parteien und Kandidaten durch den Zufluss von Ressourcen (Geld, einseitige Berichterstattung), ein falscher Zuschnitt der Wahlkreise, interessenverzerrende Wahlverfahren oder die Behinderung der parlamentarischen Arbeit. Auch die Wahlbeteiligung gilt als Indikator. Diese ist in Deutschland trotz aller Phänomene der Politikverdrossenheit weiterhin vergleichsweise hoch, allerdings geht sie wie in vielen OECD-Staaten insgesamt zurück. Die Parteien verlieren seit vielen Jahren Mitglieder. Aber selbst, wenn das nicht der Fall wäre, lassen sich Willensbildungsprozesse in Parteien nur mit großen Abstrichen als demokratisch bezeichnen. Es ist ja bedenklich, dass Parteien eines der wichtigsten Willensbildungsorgane der Demokratie darstellen, aber tatsächlich nur ein Bruchteil der Mitglieder und gar der Bevölkerung an ihren Diskussionen teilhaben. Es kommt zu erheblichen Repräsentationsdefiziten. ArbeiterInnen, Frauen, Migrantinnen sind schlecht vertreten. Oftmals schaffen es eher rechtspopulistische Parteien Teile der unteren Klassen anzusprechen. Die berechtigte Kritik an denen 'da oben' wird mit anti-demokratischen Affekten verknüpft. Doch gerade rechtspopulistische Parteien haben sich immer wieder als äußerst korrupt erwiesen.

Parteien, erschreckt von Mitgliederschwund und schlechten Wahlergebnissen, haben seit längerem und mit unterschiedlicher Konsequenz versucht, die Defizite an Engagement und Partizipation zu beheben: offene Mitgliederversammlungen, offene
Wahllisten, Quoten für Frauen und Jüngere. Bei den Piraten gab es Diskussionen über einen Internetbasierten Parteitag, das wurde abgelehnt. Auch Versuche in der Partei Die Linke, einen virtuellen Landesverband zu gründen, sind bislang gescheitert. Es bilden sich zahlreiche Protestparteien, manche verstehen sich als anti-staatlich, manche als Anti-ParteiParteien. Das gilt für rechtspopulistische Parteien wie die Freiheitlichen in Österreich, für die SVP in der Schweiz, die Lega Nord in Italien. Auf der demokratischbürgerrechtlichen Seite galten die Grünen lange Zeit als Anti-Parteien-Partei, die sich aber mittlerweile gut ins politische System eingefügt haben, aber immer noch den Ruf des Unkonventionellen genießen. In jüngerer Zeit brachten die Piraten die Parteienlandschaft und das Selbstbewusstsein der etablierten politischen Kräfte durcheinander und stellten deren Selbstverständnis auf die Probe, indem sie neue Formen der direkten Demokratie versprechen. Die Erwartungen an solche Formen sind groß, auch mit Volksentscheiden werden große Hoffnungen verknüpft, obwohl die konkreten Beispiele von direkter Demokratie eher enttäuschend sind.

Die Krise der Demokratie lässt sich auch am Zustand der politischen Kultur eines Landes ablesen: der Zustand der Bürgerrechte (Demonstrationsfreiheit), die Meinungsfreiheit (z.B. das Recht, Religion öffentlich zu kritisieren), die Sicherheit von JournalistInnen, die Rechte von Lohnabhängigen, sich in Gewerkschaften zu organisieren und zu streiken. Es gehört dazu, dass Schwule und Lesben ihre sexuelle Orientierung frei leben können, dass Abtreibung ohne strafrechtliche oder moralische Sanktionen bleibt oder Rassismus und Antisemitismus nicht offen oder stillschweigend akzeptiert wird. Auch die Freiheit der Wissenschaften, der Forschung 
und Lehre müssen dazu gezählt werden. In manchen dieser Hinsichten hat sich das $\mathrm{Maß}$ an Freiheiten in der Bundesrepublik vergrößert - Schwule werden nicht offen diskriminiert oder gar verfolgt, Journalisten nicht ermordet. Aber es gibt dennoch zahlreiche Einschränkungen: Gewerkschaftern werden Hindernisse in den Weg gelegt und immer wieder werden sie und ihre Aktivitäten in der Öffentlichkeit delegitimiert; Journalisten werden behindert oder ausspioniert, ihre Artikel nicht gedruckt, ihre Recherchen nicht gesendet. Bürgerrechte bleiben vielfach prekär: so werden Antifaschisten mit Demonstrationsverboten überzogen und mit polizeilicher Gewalt konfrontiert oder DemonstrantInnen von der Polizei rechtswidrig von Kundgebungsorten verbannt. Es gehören zur demokratischen politischen Kultur weitere Rechte, die durch das Planungsrecht oder das Baurecht. Werden Bürger informiert, haben sie Zeit, sich in Planungsvorhaben einzubringen oder diese mit guten Argumenten zu verhindern, sind Fragen, an denen sich die Beurteilung der Demokratie entscheiden und der Protest entzünden kann.

Mit der großen Krise gingen zahlreiche große Protest- und Demokratiebewegungen einher. Es sind insbesondere die sozialen Bewegungen in den arabischen Ländern, M15 in Spanien, Occupy in den USA, die Proteste in Israel, in England oder Kanada, in Bulgarien, Slowenien oder in Russland zu nennen. In Deutschland waren es die Proteste gegen Stuttgart 21 oder gegen die Endlagerung in Gorleben, neuerdings gegen die Stromtrassen oder Fracking, die die Aufmerksamkeit der Öffentlichkeit mobilisieren. Immer wieder wird der Mangel an Demokratie und Beteiligung beklagt und kritisiert, dass in der repräsentativen Demokratie die Interessen der Bevölkerung nicht vertreten sind, sondern sich die Politik allein an den
Interessen der Mächtigen und den „Märkten" ausrichtet.

Die Ungewissheit über die Demokratie hat Forderungen nach direkter Demokratie gestärkt. Die Themen Demokratie und Öffentlichkeit, Parteien und Parlamentarismus sind in den letzten Jahren verstärkt Gegenstand politischer Auseinandersetzungen geworden. Auch in der politischen Theorie findet eine regelrechte Renaissance der Demokratietheorie statt.

Der Zustand der Demokratie wie auch die kritischen Diagnosen geben Anlass, über das Verhältnis der Linken zur parlamentarischen Demokratie und ihren Institutionen ebenso wie über die kritischen Begriffe und Instrumente, die in den vergangenen Jahrzehnten entwickelt wurden, nachzudenken. Eines der wichtigsten Motive in der kritischen Gesellschaftstheorie war die Feststellung, dass politische und insbesondere parlamentarische Prozesse nicht oder nur unter bestimmten Bedingungen die grundlegenden Bedingungen der gesellschaftlichen Entwicklung, also die Prozesse der Kapitalverwertung und die damit verbundenen sozialen Auseinandersetzungen gestalten können. Deswegen kann eine kritische Analyse nicht bei den immanenten Problemen der parlamentarischen Institutionen stehen bleiben. Mehr noch stellt sich die Frage, die in der kritischen, materialistischen Theorie immer wieder aufgeworfen wurde, welche Bedeutung Parlament und Parteien für die Aufrechterhaltung und Umsetzung von Herrschaft selbst haben. Anders als oftmals vertreten, sind diese Institutionen keine neutralen politischen Formen, sondern organisieren Interessen und Willensbildungsprozesse auf spezifische Weise. Interessen von unten kommen nun einer stark veränderten Form durch den Prozess der öffentlichen und parlamentszentrierten Diskussion und Entscheidungsfindung. So 
haben Parlament und Parteien eine widersprüchliche Wirkung. Sie gewährleisten bestimmte Freiheiten, gleichzeitig aber tragen sie dazu bei, dass die Gesellschaft enteignet wird, sich selbstbestimmt zu koordinieren. Es kommt zu der von Marx schon ausführlich beschriebenen Aufspaltung in partikulare und allgemeine Interessen. Parlamentarische Demokratie trägt zu dieser Spaltung bei. Aus einer kritischen Sicht ist immer zu prüfen, ob und wieweit parlamentarische Prozesse es überhaupt erlauben, emanzipatorische Ziele zu verfolgen und umzusetzen.

Alle diese Fragen haben uns bei der Planung des vorliegenden Heftes beschäftigt. Wir hatten die Erwartung, dass zu vielen dieser Aspekte Texte eingereicht würden. Wir waren jedoch überrascht, feststellen zu müssen, dass dies nicht im vermuteten Umfang der Fall war. Kritische und linke Diskussionen über parlamentarische Demokratie haben gegenwärtig, so ist unser Eindruck, einen anderen Schwerpunkt. Eher geht es um Fragen der Selbstorganisation - und offensichtlich spielen dabei die Erfahrungen in Zusammenhängen sozialer Bewegungen und mit der Partei Die Linke eine wichtige Rolle. Diskutiert werden Fragen der Organisation, also der kollektiven Handlungsfähigkeit und der demokratischen Willensbildung innerhalb von Organisationen und Bewegungen. Die historischen Erfahrungen mit autoritären Umgangsweisen führen offensichtlich zu einer Suche nach neuen organisatorischen Formen, die demokratische Binnenverhältnisse ermöglichen.

Alex Demirović stellt die Diskussionen über eine Krise der Demokratie und autoritäre Entwicklungen in den Horizont der „multiplen“ Krise des gegenwärtigen Kapitalismus und der Entstehung neuer oppositioneller Akteure und der Debatte um eine radikaldemokratische Erneuerung. Anne
Steckner wendet sich der marxistischen Parteiendiskussion zu. Sie versucht unter Rückgriff auf Gramscis Hegemoniekonzept genauer zu bestimmen welche Rolle die Parteien bei der Herstellung und Durchsetzung von Konsens spielen. AnnKathrin Krug und Jacob Graf sehen in ihrem Artikel bei Gramsci und Luxemburg sich einander ergänzende Anknüpfungspunkte für aktuelle Organisationsdebatte. Allerdings, so Krug und Graf, sollten viel stärker als dies in den meisten Diskussionsbeiträgen geschieht, die gesellschaftlichen und politischen Verhältnisse berücksichtigt werden, auf die sich die Analysen von Luxemburg und Gramsci beziehen. Mit dem Begriff der „Mosaik-Linken“ setzt sich Alexander Neupert kritisch auseinander und diskutiert die Bedingungen, unter denen die einzelnen Mosaiksteinchen tatsächlich kooperieren können: gemeinsame Interessen reichen dafür nicht aus. Mit Strömungen des Rechtspopulismus, die inzwischen auch mehr direkte Demokratie fordern, beschäftigt sich Holger Oppenhäuser. Dabei wird deutlich, dass die Forderung nach direkter Demokratie keineswegs frei von Ambivalenzen ist. Das auf vermehrte Proteste gegen große Infrastrukturprojekte mit einer stärkeren Bürgerbeteiligung reagiert wird, mag zunächst als Fortschritt erscheinen. Thomas Wagner macht in seinem Beitrag aber deutlich, dass es sich dabei auch um eine "Mitmachfalle" handeln kann, dass eine größere Partizipation die Ausübung von Herrschaft auch effizienter machen kann.

In einem Einspruch setzt sich Alex Demirović kritisch mit dem neuesten Buch von Wolfgang Streeck auseinander: es mache unfreiwillig deutlich, wie sehr das sozialdemokratische Projekt gescheitert sei, aber auch wie unfähig die sozialdemokratischen Intellektuellen seien, dieses Scheitern zu reflektieren. 
Außerhalb des Schwerpunkts beschäftigen sich Jannis Milios und Dimitris Sotiropoulos mit der Eurokrise. Sie zeigen auf, dass der Euro nicht einfach nur eine Währung ist, sondern ein Mechanismus, der von einzelnen Ländern Anpassungsprozesse erfordert, die letztlich nur erreicht werden können, indem die Arbeitsbedingungen und die Masseneinkommen den Erfordernissen des Kapitals unterworfen. Insofern muss die Konstruktion des Euro wie auch die gegenwärtige Krise nicht als Ergebnis einer unzureichenden Politik, sondern als Bestandteil des Klassenkampfes aufgefasst werden. Albert Scherr setzt sich schließlich mit der von migrationspolitischen Gruppen erhobenen Forderung nach „offenen Grenzen" und der nicht so ganz einfachen Kritik am Nationalismus auseinander. Bernd Röttger erinnert an den im Februar verstorbenen Gilbert Ziebura, der auch für die PROKLA ein geschätzter Autor und Gesprächspartner war

Als Gastredakteur hat Ingo Stützle am Schwerpunkt dieses Heftes mitgewirkt. Wir danken ihm ganz herzlich für seine Ideen und sein Engagement.

\section{PROKLA 172: Gesellschaftstheorie III: Zeitdiagnosen (September 2013)}

Bereits 2011 und 2012 sind zwei PROKLA-Hefte erschienen, die grundsätzliche Fragen der Gesellschaftstheorie behandelten. In diesem dritten Heft sollen aktualitätsbezogene Ansätze und Zeitdiagnosen im Mittelpunkt stehen. Dies betrifft die Bestimmung der Gesellschaft selbst: Ist sie eine Wissens-, eine Dienstleistungsgesellschaft? Kann sie als kognitiver Kapitalismus verstanden werden? Ist sie neoliberal, allein von der Logik des Marktes bestimmt, oder auch noch von Religion und ständischen Formen geprägt? Nimmt die soziale Integration der Gesellschaft zu oder lösen sich soziale Bindungen immer weiter auf? Bedeutet mehr Integration mehr Zwang und Druck auf die Einzelnen oder gewinnen sie mehr gesellschaftlich vermittelte Handlungsfreiheit? Wird die Gesellschaft komplexer, indem immer mehr Handlungsbereiche Autonomie erlangen oder wird sie einfacher, weil es in vielen Bereichen zu einer Entdifferenzierung kommt? Beschleunigt sich die Gesellschaft immer noch weiter, kann die Zeit den Raum immer noch weiter verdichten und vernichten - worüber schon in der Mitte des 19. Jahrhunderts geklagt wurde oder gewinnen Räume heute ein neues Eigengewicht? Was bedeuten zunehmende Urbanisierung oder höhere Mobilität? Welche Folgen haben hohe Scheidungsquoten, geringe Geburtenraten, ein hoher Altersdurchschnitt? Welche Folgen für das gesellschaftliche Leben haben die Zunahme von Reichtum und Armut? Gibt es das oftmals beschworene Phänomen der Angst in breiten Bevölkerungsgruppen? Wie wirken sich gesellschaftliche Kontrolldispositive aus? Wichtige Ansätze und Begriffe der aktuellen Debatten über die kapitalistische Gesellschaft sollen in ihrer zeitdiagnostischen Bedeutung ausgeleuchtet und in kapitalismuskritischer Perspektive analysiert werden. 